\title{
Historische Etymologien und Wortgeschichten in historischen Bedeutungswörterbüchern
}

$\begin{array}{llll}1 & \text { Sprache und Sprachwissen } & 4 & \text { Methodische Überlegungen } \\ 2 & \begin{array}{l}\text { Sprache, Sprachwissen und Sprachwandel } \\ \text { im historischen Bedeutungswörterbuch }\end{array} & 5 & \text { Beispiele } \\ 3 & \begin{array}{l}\text { Historische Wortgeschichten im } \\ \text { historischen Bedeutungswörterbuch }\end{array} & & \text { Literatur }\end{array}$

\section{Sprache und Sprachwissen}

Wissen über Sprache überhaupt gehört ebenso zur sprachlichen Kompetenz wie das Wissen um die Möglichkeiten und Restriktionen der Wortstellung im Satz, um die korrekte Betonung von Frage- und Aussagesätzen, um die Präpositionalkasus oder um Wortbedeutungen. Genauso freimütig wie etwa falsches Schreiben korrigiert und falsches Sprechen kritisiert wird, wird - im Zusammenhang mit solcher Kritik oder abseits von ihr in Diskussionen ohne solche konkreten Anlässe - über Sprache reflektiert: „Also ich verstehe unter Sprache ...", „Aber Sprache ist doch nicht ...". Sprache ist ohne und abseits von Reflexion über Sprache nicht denkbar. Leicht ließe sich anhand einer ganzen Reihe von Beispielen zeigen, wie das Wissen um die Sprache den Umgang mit ihr beeinflusst und wie wiederum der Umgang mit der Sprache ihre Wirkung auf das Wissen um dieselbe ausübt.

Solches Wissen um Sprache kann in verschiedenen Formen zutage treten: etwa als Regelwissen in der Rechtschreibung, als die Fähigkeit, die Herkunft eines Dialektsprechers zu erkennen oder als Korrektur einer falschen Aussprache im Fremdsprachenunterricht; es kann in Witze, Parodien oder in Hetzreden Eingang finden.

\section{Sprache, Sprachwissen und Sprachwandel im historischen Bedeutungswörterbuch}

Vor diesem Hintergrund wird deutlich, dass das Verhältnis zwischen Sprache und Sprachwissen bei der Betrachtung von sprachlichem Wandel besondere Beachtung erfordert: Sprachwandel ist auch immer Wandel des Wissens um Sprache, ebenso wie einerseits das Wissen um Sprache vom Sprachwandel beeinflusst werden, andererseits dieses wiederum 
auf den Sprachwandel einwirken kann. Ein typisches Beispiel hierfür wäre die Wirkung von Sprachkritik. ${ }^{1}$

Fragen des Sprachwandels finden in unterschiedlichsten Formen Eingang in Darstellungen vergangener Sprachstufen. Darunter sind traditionell auch die Wörterbücher. Aufgrund ihrer speziellen formalen Charakteristika - deren traditionelle Verdichtung, Knappheit und Strenge sich in jüngerer Zeit in begrüßenswerter Weise zu lockern beginnen (vgl. z.B. WelZIG 1999) - sind der Aufnahme von Daten zu den eingangs dargestellten Zusammenhängen jedoch recht enge Grenzen gesetzt. Dennoch kann auch - und vielleicht sogar gerade - ein historisches Wörterbuch den Zusammenhängen von Sprache und Sprachwissen auf verschiedenste Weise Rechnung tragen. Schon seinem Wesen gemäß ist das Wörterbuch eng mit dem Wissen um Sprache verknüpft: Der ureigenste Zweck eines (Bedeutungs)Wörterbuchs ist die Erklärung, typischerweise die Erklärung von Wortbedeutungen. Diese Erklärungsleistung ist auf zweifache Weise an das Wissen von Sprechern um Sprache gebunden: zunächst und primär an das Wissen des Lexikographen über das Explanandum (das durch verschiedenste Faktoren eingeschränkt sein kann: zeitliche oder finanzielle Begrenztheit, Forschungslücken der Lexikologie, Platzmangel, Thematik des Wörterbuchs, sowie das nicht zu lösende Problem der immer ungenügenden Kompetenz des Lexikographen in Bezug auf die Sprache der historischen Zeitstufe) und sekundär an das Wissen des Wörterbuchbenutzers. Schließlich erklärt der Lexikograph die Wortbedeutungen immer jemandem. Über das Wissen des Benutzers wird der Lexikograph in den meisten Fällen zwar nur Vermutungen anstellen; doch basiert ein solcher ,educated guess“ natürlich auf bestimmten, meist allerdings nur wenig fundierten Vorstellungen von der avisierten Zielgruppe, die wiederum auf den Erfahrungen des Lexikographen beruhen.

Dies gilt natürlich für alle Wörterbuchtypen; bei lexikographischen Unternehmungen, die auf die Erklärung historischer Sprachzustände ausgerichtet sind, hat dies jedoch ganz spezifische Auswirkungen. Ein solches Wörterbuch verzeichnet ja „historische“ Wörter eventuell in ihren „historischen“ Formen (Schreibungen, Aussprachen), in „historischen“ Kontexten (repräsentiert durch Belege) und in „historischen“ Verwendungen (Bedeutungen). Vor allem die Bedeutungsbeschreibung ist im historischen Wörterbuch ein neuralgischer Punkt. Anders als die Angabe historischer Schreibungen, die der Lexikograph nur versammeln und organisieren muss, ${ }^{2}$ ist die Bedeutungsangabe ausschließlich ein Konstrukt, das auf der Interpretation des Lexikographen beruht. Dies allein macht jedoch noch nicht den Unterschied zum gegenwartssprachlichen Wörterbuch aus. Dieser besteht vielmehr darin, dass der Lexikograph hier anders als der Kollege keinerlei aktive Sprachkompetenz in Bezug auf die in Frage stehende Sprachstufe besitzt. Das Zusammenspiel unterschiedlicher sprachlicher Kompetenzen ist hier äußerst komplex: Genau besehen erklärt er nicht seine Auffassung einer historischen Wortbedeutung, sondern seine Auffassung der Auffassung der damaligen Sprecher von dieser Wortbedeutung, die ihm dann auch noch nur in Ausschnitten, d.h. in konkreten Verwendungen vor Augen tritt; das Ganze wird zusätzlich durch die Benutzung weiterer (historischer) Wörterbücher verkompliziert.

1 Man denke etwa an das Verschwinden des Wortes Fräulein aus nahezu allen seiner ehemals typischen Gebrauchssituationen.

2 Auf die dabei und in Bezug auf die Lemmatisierung auftretenden Probleme soll in diesem $\mathrm{Zu}$ sammenhang nicht eingegangen werden. 
Ein historisches Wörterbuch ist also auf eine ganz spezielle Weise in das Verhältnis zwischen Sprache und sprachlichem Wissen eingebunden. Alle Angaben, die der Lexikograph macht, sind Ausdruck dieser speziellen Geartetheit des Zusammentreffens von Sprache und Sprachwissen im historischen Wörterbuch: Die Grammatikangabe zu hochmuot und lancmuot in einem Wörterbuch zur mittelhochdeutschen Sprache muss natürlich historisch korrekt „f.“ lauten. Dies ist schlichte Abbildung belegbarer historischer sprachlicher Verhältnisse. Problematisch kann dies werden, wenn durch solche Angaben sachlich falsche Daten Eingang ins Wörterbuch finden: Berühmt ist der Artikel Blindschleiche im DWB. JАСОВ GRIMм hat bekanntlich als Erklärung für dieses Wort formuliert: ,eine blinde, giftige schlange“ (DWB Bd. II, Sp. 126). Wir wissen heute zwar, dass Blindschleichen sehende, ungiftige Eidechsen sind; aber ebenso liegt auf der Hand, dass in einem Wörterbuch, das heute zu einer Epoche erstellt würde, in der man Blindschleichen für blinde, giftige Schlangen hielt, genau diese Bedeutungserklärung wieder erscheinen müsste. Dies geschähe zwar wider besseres Wissen, aber eben wider besseres rezentes Wissen -, von dem im Übrigen auch nicht sicher ist, ob es nicht nach weiteren 147 Jahren als Beispiel in einem Beitrag ähnlicher Thematik Verwendung findet. Ein Lexikograph, der sich zur Aufgabe gemacht hat, die Sprache um 1800 zu erfassen, muss daher die Gotik als deutsche Baukunst beschreiben oder zumindest die entsprechende Bedeutungsangabe um eine Bemerkung wie etwa „Die in dieser Zeit entstandene Baukunst wurde weithin fälschlich als charakteristisch deutsch angesehen“ ergänzen, auch wenn heute die Herkunft der Gotik aus Frankreich als historisch erkannt ist. Andernfalls würde sein Artikel bei einem nachschlagenden Benutzer nur Verwirrung stiften. Denn dieser hat unter Umständen eine Textstelle vor sich, in der die Gotik gegen die französische Architektur ins Feld geführt wird und wundert sich über diese Wortverwendung.

Wir können also, ausgehend von diesen Überlegungen, lexikographische Angaben im Hinblick auf den Umgang mit historiolingualem bzw. historiolinguistischem Wissen unterscheiden: Es gibt zum einen solche Angaben, die vergleichsweise eindeutig und problemlos belegte historische Zustände schlicht beschreiben. Dazu gehören z.B. einige Grammatikangaben $^{3}$ und die Angaben zur Graphie. Diesen stehen solche Angaben gegenüber, in denen ein - relativ zum Wissensstand der Bearbeitungszeit aus diesem Grund nur unter Vorbehalt zu verstehendes - Wissen um Irrtümer der Zeit, um „richtig“ und „falsch“ eine Rolle spielten. Beide Sorten von Angaben können eine weiterführende Kommentierung erfordern, die jedoch jeweils unterschiedlichen Charakters sein muss.

\section{Historische Wortgeschichten im historischen Bedeutungswörterbuch}

Nun könnte eine solche Unterscheidung trivial wirken, gäbe es nicht eine weitere Sorte von Angaben, die die Problematik dieser Situation deutlich macht. Eine besondere Rolle spielen in diesem Zusammenhang nämlich Angaben zu Etymologie und Wortgeschichte.

3 Detailprobleme wie z.B. die Schwierigkeiten der Genusbestimmung in einigen syntaktischen Kontexten seien hier außer Acht gelassen. 
Das Interesse an etymologischen Fragen zu allen Zeiten und insbesondere der Lexikographen - verbunden mit den unterschiedlichsten Zielsetzungen ${ }^{4}-$ ist bekannt und bedarf hier keiner Darstellung. ${ }^{5}$ Ebenso groß war und ist die Verbreitung etymologischen Wissens - jedenfalls in bestimmten Kreisen und in Bereichen, in denen Fragen der Etymologie eine bestimmte, über sie selbst hinausweisende Bedeutung annehmen konnten. ${ }^{6}$ So war in früheren Zeiten die etymologische Verbindung von Gott und gut, die sich zur Stützung theologi-

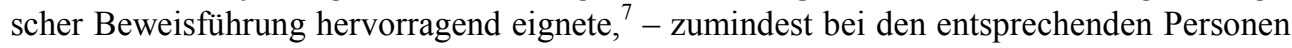
- sicher nicht weniger gut bekannt als die heute weithin für korrekt gehaltene Verwandtschaft des Wortes mit gießen (vgl. KLuge 2002, 366). Ebenso ist der Hinweis „Kunst kommt von können“ noch heute ein beliebtes Argument in Diskussionen um „moderne“ Kunst.

Der Zusammenhang mit unseren bisherigen Überlegungen ist klar: Genau so wie Wissen um Sprache überhaupt mit der Sprache verwoben ist, so ist auch etymologisches Wissen eng mit der Sprache verbunden.

Nun ist jedoch auch bekannt, dass die Etymologie bis ins 19. Jahrhundert hinein eine äußerst spekulative Wissenschaft war. ${ }^{8}$ Vieles an etymologischem Wissen, das in Umlauf war (und zum Teil weiterhin ist), entsprach nicht dem heutigen Wissensstand, ganz zu schweigen von der sprachhistorischen Realität. Dies ist von umso größerer Tragweite, als dieses Wissen keine ausschließlich sprachlichen Konsequenzen hat. KURT BALDINGER zeigt anhand des folgenden Beispiels, dass etymologisches Wissen, so sehr es sich auch später als falsch erwiesen haben mag, erhebliche Auswirkungen auf die Interpretation oder gar auf die Schaffung von „Realität“ haben kann. Er weist darauf hin, dass frz. batiste noch in BlOCH-WARTBURG ${ }^{4} 1964$ als auf Baptiste, den „nom du premier fabricant de ce tissu, qui vécut au $\mathrm{XIII}^{\mathrm{e}}$ s., à Cambrai ${ }^{\text {“9 }}$ zurückgehend interpretiert wird und führt weiter aus:

„Manfred Höfler hat nachgewiesen, daß batiste in Wirklichkeit von battre abgeleitet wurde, der erste Fabrikant wurde offensichtlich von der Phantasie des Volkes geschaffen und sogar mit einem Denkmal geehrt: „Die Volksetymologie beschränkt sich nicht darauf, eine Wortform unter dem Druck eines sekundär hineingedeuteten Wortes umzugestalten, die neue Form kann auch ihrerseits kulturhistorisch aktiv werden und selbst bis zur Errichtung eines Denkmals führen, zu Ehren eines Mannes, der nie gelebt hat" (Manfred Höfler, Fr. batiste und das volksetymologische

4 Vgl. hierzu beispielweise PfeIFER 1984, 233-238.

5 Auch heute ist das Interesse an etymologischen Fragestellungen ungebrochen. So gehört die Frage nach der Etymologie eines Wortes erfahrungsgemäß zu den häufigsten Fragen des Laien an den Linguisten, und oft wird die Etymologie bemüht, wenn in Auseinandersetzungen bestimmte Wahrheiten formuliert werden sollen (,Kunst kommt von können“). Vgl. hierzu GaUger 1994, 23-39. Der Verkaufserfolg von etymologischen Wörterbüchern einerseits (z.B. KLUGE 2002.) und für ein breiteres Lesepublikum bestimmten Wortgeschichten andererseits (z.B.: BARTELS 1996) bestätigt diesen Eindruck. Vgl. hierzu auch die Vorbemerkungen YaKov MALKIELS zu seinem Aufsatz „Das etymologische Wörterbuch von Informanten- und Korpussprachen“ 1990, $1323-1334$.

6 Vgl. hierzu etwa WunderLi 1994a, darin seinen Beitrag „Sprachursprungstheorien in der Romania“, 179-205, in dem er eine ganze Reihe von Beispielen für das Stützen einer Argumentation auf (vermeintliche) etymologische Verwandtschaft anführt.

7 Vgl. hierzu den Hinweis von Renate Bebermeyer im Zusammenhang mit der Frage nach dem Verhältnis von „Volk“ und „Gelehrten“ im Rahmen der Volksetymologie in: BEBERMEYER 1974, 174.

8 Vgl. hierzu z.B. Pfeifer 1984 und Abaev 1980, 35.

9 Bloch/von Wartburg 1964, 62. Hinweis bei: Baldinger 1973, 34. 
Denkmal, ZrP 80, 1964, S. 455-464). Auch in diesem Falle handelte es sich um zwei verschiedene Wortfamilien [...].“ (BALDINGER 1973, 34f.)

Noch heute findet sich, Höflers Untersuchung zum Trotz, auf der website http: / / www . cambresis.cci.fr folgender Satz: „Au Moyen-Age, un tisserand appelé Baptiste Cambray inventa la „batiste“, une fine toile de lin très appréciée tout au long des siècles. “" ${ }^{10}$

Doch solche Erscheinungen sind nicht auf die jüngere Vergangenheit beschränkt: Als weiteres Beispiel für die Nutzbarmachung solcher heute als falsch geltender Etymologien mag die Verknüpfung der Wörter theodiscus bzw. teutonicus mit dem ägyptischen Gottesnamen Theut, dem Namen des mythischen Erfinders der Schrift, im späten Mittelalter dienen. ${ }^{11}$ Hannes KäSTNER schildert damit keinen Einzelfall: Tatsächlich wurden solche Herleitungen oft dazu verwendet, den Anspruch auf bestimmte Vorrechte zu stützen. So entnahm man etwa dem römischen Namen der Stadt Xanten, Colonia Ulpia Trajana, dass diese eine Gründung der auf der Flucht befindlichen Trojaner gewesen sei, die schließlich unter der Führung Frankos, des Stammvaters der Franken, an den Niederrhein gelangt seien. Diese Sage, im Kern bereits in den frühmittelalterlichen „Gesta Regum Francorum“ vorhanden, wurde in der Folgezeit ausgebaut und galt lange als tatsächliche Frühgeschichte der Franken. Mit ganz ähnlichen Mitteln versuchten auch andere Städte, ihre Ansprüche zu stützen, so z.B. Reims, das natürlich von niemand anderem als von REMUs gegründet worden sein konnte. ${ }^{12}$

Auch und vielleicht gerade die weniger spektakulären Fälle zeigen, wie wichtig es ist, jede Form von etymologischer (Re)interpretation auch in Bezug auf ihre Auswirkungen auf die „Vorstellungen des Menschen“ (vgl. den Titel eines bereits erwähnten Textes von Kurt Baldinger) nicht zu unterschätzen:

„Wer z.B. grâ smücke ,die grau Geschmückte“ in Grasmücke ,Gras-Mücke’ umdeutet, der hat anders assoziiert: der neue Name ist nicht mehr von dem damit benannten Tier her motiviert $[\ldots]^{\star 13}$

Natürlich kann aber dann die Auffassung vom Tier von der Neuinterpretation seines Namens her beeinflusst werden: ${ }^{14}$ So ,,ist man oft der Versuchung erlegen, die Bedeutung dieser nicht auf die Umstände ihrer Entstehung weisenden, vermittelten Bezeichnungen und die Vorstellung, die sie nun sekundär bieten, mit dem bezeichneten Tier in direkte Verbindung zu bringen, dieses dann zum Träger objektiv oder subjektiv entsprechender Eigenschaften zu machen und daraus endlich den Namen zu erklären.“ (BAUMANN 1967, 29). Denn „Vorstellungen [...] pflegt man mit empfangenen Mitteilungen zu verbinden; wenn sie die sachliche Seite des Wortes nicht bietet, weil das ,Mitgeteilte“ (= das gemeinte Tier) nicht bekannt ist, dann kann seine sprachliche Seite doch leicht als Aufhänger dazu dienen.“ (BAUMANN 1967, 35).

10 http://www.cambresis.cci.fr/DEV-ind-tex-cam-his.htm, Stand 17. 1. 2007. Copyright der Page von 2005.

11 Vgl. hierzu KäSTNER 1991, 68-97.

12 Vgl. hierzu WeIsGerber 1957, $83 \mathrm{ff}$.

13 Baumann 1967, 21. Interessant ist nebenbei in diesem Zusammenhang die Trennung: „Amtlich“ ist auch nach der Rechtschreibreform die etymologisch widersinnige Trennung nach $<\mathrm{s}>$.

14 Wie etwa in diesem Fall, da die geringe Größe der verschiedenen Grasmückenarten (unter $13 \mathrm{~cm}$ ) sie für den Vergleich mit einer Mücke geradezu prädestiniert. 
Ganz gleich, wie man solche „schöpferische Mißverständnisse“15 einordnen will und von wem diese überholten Etymologien vorgeschlagen wurden (ob von Etymologen, Pastoren, Beamten, oder eben vom „Volk ${ }^{\text {“16 }}$ ), für das Wörterbuch ergibt sich unter Umständen die Notwendigkeit, diese aufzugreifen, da diese, wie wir gesehen haben, auf die Realitätsauffassung der Menschen und ihre sprachliche Erfassung ihre Auswirkungen haben kann. Die Einteilung in Etymologie und Volksetymologie ${ }^{17}$ spielt dabei nur eine untergeordnete Rolle - auch wenn vor allem in Arbeiten zu letzterer, unter Stichworten wie Remotivation,

15 So genannt von Kurt BALDinger in Anlehnung an den Titel eines Beitrags von Johann KnobLOCH. Weitere Beispiele für solche schöpferische Missverständnisse finden sich u.a. bei WeIsGERBER 1957, im Rahmen des Kapitels „Sprachliche Wirkungen in Sitte und Volksbrauch“, 5994 sowie in BALDINGER 1973, passim. Er berichtet auch den folgenden Fall:

„Der Ohrkneiper (Ohrwurm), frz. perce-oreille, it. forfecchia, tenaglia wurde so genannt, weil sein gegabelter Schwanz an ein Goldschmiedzänglein erinnerte, mit dem man das Ohrläppchen durchbohrte, in das ein Ohrring eingeführt werden sollte (früher eine viel weiter verbreitete Sitte). Die Entmetaphorisierung führte dazu, daß man zu glauben begann, der Ohrkneiper krieche in das Ohr hinein (sekundär z.B. schweizerdt. Ohregrübler; wall. mousse-è-l'oreille zu nam. moussî „s'introduire, pénétrer quelque part“ FEW 63 3 , 193b; 196a), durchlöchere das Trommelfell und verstecke sich im inneren Ohr.“ (a.a.O, S. 30).

Die Quelle für dieses Beispiel ist sicherlich die Arbeit von Elfriede Werz, „Der Ohrwurm“. Diss. Marburg 1945.

16 Vgl. hierzu Olschansky 1996, 119, v.a. Anm. 63 sowie S. 154-165, ebenda. Der Terminus Volksetymologie geht auf den Aufsatz „Ueber deutsche volksetymologie“ von ERNST FöRSTEMANN (1852) zurück. FöRSTEMANN ist der erste, der diese Form von Remotivation mit dem ,ungebildeten Volksgeist" in Verbindung bringt und sie ausdrücklich als unwissenschaftlich beschreibt. Vgl. FöRSTEMANN 1852, 2. In jüngerer Zeit scheint sich eine Tendenz in die entgegengesetzte Richtung abzuzeichnen: nicht nur wird die Volksetymologie als vom Bildungsstand unabhängig beschrieben, sie wird sogar explizit den „,members of the literate culture even up to the most highly sophisticated journalists, historians and literary artists“ zugerechnet (so von WILLIAM KIRWIN in seinem damit letztlich inkonsequent „Folk Etymology: Remarks on Linguistic Problem-Solving and who does it" benannten Artikel in: Lore and Language IV (2) 1985, 18-24, Zitat S. 19). In diesem Zusammenhang spielt auch das bekannte Phänomen der volksetymologischen Übersetzung eine wichtige, weil bestätigende Rolle (vgl. etwa das bekannte Beispiel Vielfraß).

Tatsächlich ist der Urheber einer „Volksetymologie“ nicht immer unbekannt. So betont Peter von Polenz, Volksetymologien seien „oft eher alte Gelehrten-, Pastoren- oder Beamtenetymologien“. Vgl. hierzu Von Polenz 1980, 171.

17 KIRWIN etwa unterscheidet folk etymology, als ,re-formation of a strangely pronounced or spelled form with the result that the new term makes plausible sense" von false, or pseudo, etymologies als ,an expressed justification or explanation of the new term, when it is first used or by other commentators at a later time“, worunter auch die gelehrten, aber eben sprachhistorisch falschen, Etymologien zu rechnen wären (KIRwIN, ebenda, 22). Eine ähnliche Unterscheidung trifft auch MAX DeEg, wenn er sagt, daß gelehrte Etymologien einen „Etymologisierungsprozeß vor Augen führen, während die Volksetymologie das fertige, schon fest im Lexikon einer Sprache verwurzelte Produkt eines Prozesses darstellt. Die gelehrte Etymologie hingegen ist das Produkt einer bewußten Auseinandersetzung mit dem Wort und der Versuch, zur inneren Wahrheit des Wortes vorzudringen.“(DeEg 1995, 42.)

Tatsächlich ist der Unterschied zwischen Volksetymologie und dem jeweils neusten Stand etymologischer Forschung geringer, als es zunächst den Anschein hat. Das bringt GERD ANTOs mit folgender Formulierung treffend auf den Punkt: „Nur der je aktuelle Erkenntnisstand der Etymologie kann strenggenommen die Zuschreibung „wissenschaftlich“ in Anspruch nehmen, der je überwundene ist dem Status nach nicht besser, als das, was dem Verdikt „volksetymologisch“ verfällt.“ (ANTOs 1996, 216) Vgl. hierzu auch das Beispiel Maulwurf a.a.O, S. 223. 
etymologische Reinterpretation oder Pseudosemantisierung, ${ }^{18}$ häufiger auf die hier diskutierten Fragen eingegangen wird -, da der Urheber bzw. die Qualität des entsprechenden Wissens für das Verständnis eines entsprechenden Lemmazeichens (oder eines Phrasems) weniger wichtig ist, als dessen Verbreitung bzw. Zugänglichkeit. Dementsprechend definiert HeIKe OlschansKy Volksetymologie als einen

„Vorgang, bei dem ein synchron isoliertes und als solches unmotiviertes Wort bzw. eine solche Wortkonstituente durch Anlehnung an ein lautähnliches oder (partiell) lautgleiches nicht-isoliertes bekanntes Wort (Wortfamilie) ohne Beachtung phonetisch-phonologischer und morphologischer Gesetzmäßigkeiten, in etymologischer, diachronischer Hinsicht nicht korrekt - evtl. mit lautlicher Umbildung - neu zugeordnet, somit neu bzw. sekundär motiviert, interpretiert und de-isoliert wird, wobei das Lexem, das Produkt des volksetymologischen Prozesses ist, neue morphologische, morphologisch-semantische oder semantische Deutung oder Deutbarkeit besitzt. Der sich vollziehende Anlehnungs-, Identifikations-Prozeß kann als eine Erscheinung von Analogie im weitesten Sinne gesehen werden [...].

Der Volksetymologie unterliegt entlehntes Wortmaterial, das in der Zielsprache oft per se isoliert ist, und indigenes Wortmaterial, das durch verschiedene Ursachen isoliert geworden ist; Volksetymologie betrifft Appellativa und - wahrscheinlich öfter - propriales Material, das der Tendenz zur Isolation in besonderem Maße ausgesetzt ist.“ (OLSCHANSKY 1996, 107)

Konsequenterweise fehlt in dieser Definition der Volksetymologe, was sie für unsere Zwecke - trotz einiger Inkonsistenzen, die uns hier nicht zu interessieren brauchen ${ }^{19}$ - brauchbar macht, denn sie umfasst damit alle ,in etymologischer, diachronischer Hinsicht" nicht korrekten Zuordnungen unabhängig vom Zuordnenden.

Wichtig wird ein etymologischer Irrtum nun vor allem dann, wenn er, wie oben anhand der Beispiele gezeigt, verbreitet und dann aufgegriffen wird. RENATE BEBERMEYER weist darauf hin, dass die Rolle des Gelehrten im Rahmen volksetymologischer Vorgänge vor allem dann Gewicht bekommt, wenn er ,eine wirkliche [d.h. volksetymologische, D.B.] Umdeutung als echte Etymologie verteidigt“ (BEBERMEYER 1974, 175) und führt in diesem Zusammenhang einige interessante Beispiele an. Geht eine solche „Verteidigung“, bzw. eine falsche etymologische Zuordnung z.B. in ein etymologisches Wörterbuch ein, kann dies einerseits ein Dokument der oben beschriebenen realitäts- oder zumindest sprachgebrauchprägenden Wirkung einer solchen Umdeutung sein; zudem wird sie damit für ein breites Publikum nachschlagbar, und kann damit andererseits umso leichter eine solche Wirkung entfalten.

Heike Olschanskys Definition impliziert bereits eine Unterscheidung verschiedener Typen von Volksetymologien. Sie greift daher in der Folge eine von HermanN PAUL gemachte Unterscheidung auf, nämlich die in ,Volksetymologie ohne ausdrucksseitige Veränderung“ und „Volksetymologie mit ausdrucksseitiger Veränderung“ (cf. OLSCHANSKY 1996, 107f.). Dass uns in erster Linie (aber nicht nur!) solche Volksetymologien interessieren, die ohne eine Veränderung der Ausdrucksseite vor sich gehen, liegt auf der Hand, da die anderen ohnehin (etwa als Verballhornungen ${ }^{20}$ ) kommentiert werden

18 Eine umfassende Zusammenstellung dieser und anderer Termini findet sich in: OlschansKY 1996, 108-114.

19 So vermisst man etwa den Bereich der Phraseme; auch wäre z.B. zu fragen, ob eine zufällig etymologisch korrekte Zuordnung zu einem Wort bzw. zu einer Wortfamilie nicht auch unter dem Begriff Volksetymologie gefasst werden müsste.

20 Ein bekanntes Beispiel wäre Kunterbunt, das in den meisten gängigen allgemeinsprachigen Wörterbüchern als Verballhornung gekennzeichnet ist (prominenteste Ausnahme ist das WDG). 
müssten. $^{21}$ Diese Erscheinungen verteilen sich auf die Kategorien 3 und 4 von OLSCHANSKYS eigener Typologie:
„Bei Volksetymologien des Typs (3) liegt keine lautlich [sic] Umbildung, sondern allein volks- etymologische Neuzuordnung auf der Grundlage von (partieller) Lautgleichheit zwischen einem isolierten Element und einem nicht-isolierten vor, wobei die (partielle) Lautgleichheit unabhängig von Volksetymologie (etwa durch lautgesetzliche Veränderungen, Gleitlaute, Assimilation und Dissimilation, Existenz (partieller) Faux Amis nach Fremdwortübernahme) entstanden ist; die volksetymologische Neuzuordnung bewirkt hier dann weiter Veränderung des Gesamtlexemin- halts (Referenzänderung).“(OLSCHANSKY 1996, 184)
„Volksetymologien des Typs (4) weisen keine lautliche Veränderung und keine volksetymolo- gisch bedingte Veränderung des Gesamtlexeminhalts (Referenz) auf. (Partielle) Lautgleichheit ei- nes isolierten Elements mit einem nicht-isolierten, die unabhängig von Volksetymologie entstan- den ist, ermöglicht hier synchrone entsprechende Identifikation, die in diachronischer Hinsicht fehlgeht, volksetymologische Neuzuordnung, ohne dass aber eine durch diese Neuzuordnung ver- ursachte Inhaltsveränderung zu konstatieren wäre.“ (OLSCHANSKY 1996, 186)

In diesem letzten Fall ist zwar nicht die „Referenz“, wohl aber sind Konnotationen des Ausdrucks betroffen, was diese Fälle zwar schwer erkennbar macht, aber, vor allem dann, wenn die Neuzuordnung konventionalisiert ist, umso wichtiger und damit umso interessanter für den Lexikographen. Ein Beispiel hierfür wäre Friedhof, mhd. vrithof (zu friten ,hegen, einfriedigen'), das heute, etymologisch falsch, aber dem (unveränderten!) semantischen Gehalt durchaus angemessen, gemeinhin zu Friede gestellt wird. Ist eine solche Erscheinung einmal entdeckt und durch einen glücklichen Beleg nachweisbar, so ist der Gewinn für den dazugehörigen Wörterbuchartikel, und damit für den Benutzer, nicht hoch genug einzuschätzen.

Zwar wäre es falsch, im Wörterbuch solche Ausdrücke zu einer Wortfamilie zu stellen, die zwar synchron von vielen als zusammengehörig betrachtet werden, diachron jedoch als unverwandt analysiert werden müssen, wie etwa Bratwurst und braten, Einöde und öde oder Eiland und $E i$, um nur einige der bekanntesten Beispiele zu nennen; ${ }^{22}$ dennoch müssen solche Erscheinungen in größerem Maße berücksichtigt werden, vor allem dann, wenn sie Auswirkungen auf die Weltsicht der Sprachbenutzer hatten oder haben konnten, wie dies

$21 \mathrm{Zu}$ einer Übersicht über verschiedene Typologisierungsvorschläge s. OLschansKy 1996, Abschnitt 2.8., v.a. 2.8.1.

22 Diesen Ansatz verteidigt Gerhard AugST in seinem Beitrag „Überlegungen zu einer synchronen etymologischen Kompetenz" (Augst 1975, 156-230), unter Berufung auf die, wenn auch inkonsistente, Praxis vor allem des WDG und anderer Wörterbucher zur Gegenwartssprache.

Zwar wird die Gegenposition, dass ein Wort nur dann korrekt gebraucht werden kann, wenn er Sprecher seine Herkunft kennt, heute nicht mehr vertreten, in abgeschwächter Form ist dieses Argument jedoch auch heute noch zu hören:

„Etymology has been made a strong feature of this dictionary because it is believed that insights into the current usage of a word can be gained from a full knowledge of the word's history and that a better understanding of language generally can be achieved from knowing how words are related to other words in English and to words in other Indo-European languages." (GURALNIK 1970, XII).

In der 9. Auflage des Deutschen Wörterbuchs von Hermann Paul ist zu lesen:

„Unserem Wörterbuch ist die Überzeugung eingeschrieben, dass Sprache verdirbt, wenn sie keine Anwälte mit historischem Gedächtnis hat: Wir sehen das Wörterbuch als historisches Spracharchiv, das Vergangenheit und Gegenwart verbindet, ja beide in der Geschichte aufhebt." (HenNE, Helmut/Heidrun KämPer-Jensen/Georg ObJartel: Zur Einführung. In: Paul 1992, XII.) 
oben anhand einiger Beispiele dargestellt wurde. Andererseits ist ebenfalls klar, dass das Referieren der neuesten Forschungsergebnisse mit dem eigentlichen Sinn und Zweck historischer Wörterbücher nicht in Einklang zu bringen ist. Wenn Blindschleiche in unserem hypothetischen Wörterbuch als „blinde, giftige Schlange“ erklärt werden muss, so muss auch konsequenterweise eine zu der in Frage stehenden Zeit bekannte „falsche“ Etymologie des Wortes im Artikel erscheinen. ${ }^{23}$ Eine Lösung kann also nur in der gegenseitigen Ergänzung beider Angaben liegen. Geschieht dies nicht, läuft das Wörterbuch Gefahr, durch diesen methodischen Mangel in Bezug auf die Beschreibung bestimmter historischer Wortgebräuche gerade durch eine gutgemeinte Fehler,,korrektur" fehlerhaft zu werden. ${ }^{24} \mathrm{Zu}$ denken ist hierbei vor allem an zwei Dinge: zum einen an die Instrumentalisierung von historischen Etymologien in Diskursen aller Art, die ausgehend von heutigem etymologischen Wissen nicht mehr verstehbar wäre, zum anderen an die meist weniger auffällige, aber darum umso wichtigere Nutzbarmachung solchen Wissens in literarischen Texten: Gerade Literaten, denen man im allgemeinen ein stark ausgeprägtes Bewusstsein für sprachliche Feinheiten unterstellen darf, können dieses in besonderer Weise ästhetisch, aber auch z.B. politisierend oder ironisierend einsetzen. In erster Linie ist dabei natürlich an figurae etymologicae zu denken, die Erkennbarkeit einer solchen ist jedoch sofort eingeschränkt, wenn sie auf eine heute nicht mehr anerkannte und damit nicht erkannte Etymologie gestützt wurde. Ein schönes Beispiel hierfür findet sich bei FRIEDRICH RÜCKERT, der intertextualisierend-etymologisierend schreibt:

„Wie erfreulich, wo so fraulich

eine Frau gebärdet sich!، ${ }^{25}$

Aber auch in anderer Weise kann ein Autor sein Wissen um etymologische Zusammenhänge einsetzen, z.B. um ein kulturelles Wissen seiner Leser abzurufen, von dem er ausgeht, dass diese darüber verfügen. In Wörterbüchern des 19. Jahrhunderts etwa findet sich häufig die Anmerkung, dass der Schlachtruf Hurra(h) in den Befreiungskriegen vom deutschen Heer aus dem Russischen ins Deutsche übernommen wurde. ${ }^{26}$ Es lässt sich zwar leicht belegen, dass das Wort bereits lange vor 1813 auch in deutschsprachigen literarischen Texten verwendet wurde, ${ }^{27}$ ein Autor des 19. Jahrhunderts konnte jedoch unter Umständen davon

23 Dass der Lexikograph dieses kommentieren kann und sogar muss, steht außer Frage, ist aber zunächst nicht Gegenstand unserer Überlegungen.

24 Als prototypisches Beispiel hierfür sei Matthias Lexers Mittelhochdeutsches HandwörTERBUCH genannt, in das, wie HeinRICH HetTrich gezeigt hat, der zu Lexers Zeiten aktuelle Stand etymologischer Forschung eingegangen ist, und nicht etwa die Etymologien IsIDors. Vgl. hierzu HetTRICh 1993, 133-140 sowie LindSAY 1911.

25 Zitiert nach: PAUL 1992, 290. In Anlehnung an mittelhochdeutsche Vorbilder, cf. GäRTNER 1996.

26 Vgl. die Angabe „Jubelruf, seit 1812 Kriegsgeschrei in mehreren Heeren“ in: Looff 1900, 377. Man hielt eine Verwandtschaft mit russisch/tatarisch urá ,schlag drein' für wahrscheinlich; dieser Schlachtruf sei von den russischen Soldaten übernommen worden und in den Befreiungskriegen zum Schlachtruf der deutschen Heere geworden: „hurrah, tatar. (urá, schlag drein) in den Freiheitskriegen von den Russen übernommener Schlachtruf;““ (GENIUS o.J., 409.)

27 Man denke nur an BÜRGERS „Hurrah!“ aus seiner „Lenore“ von 1773 (BÜRGER/HäNTZSCHEL 1987, 185, V. 158). Zudem findet sich in Matthias Lexers Mittelhochdeutschem HandwÖRTERBUCH der folgende Eintrag: „hurrâ interj. eigentl. imperat. von hurren mit angehängtem â ( $s p$. 1). hurrâ! wie er tobet, sô man in niht her enpfeht! MSH. 3,188b. vgl. urrâ, burrâ, wurrâ;,“ (LEXER 1872, Sp. 1397). 
ausgehen, dass ein Zusammenhang zwischen dem Wort und einem so bedeutsamen historischen Ereignis gewusst wurde, und das Wort entsprechend einsetzen. So heißt es bei ERNST MoRITZ ARndT: „Hurra, ihr Kamraden! Hurra, ihr Soldaten! / Hurra! ist Franzosen ein tödlicher Klang!“ (ARndT/LefFson 1912, 102). Und noch im 20. Jahrhundert klagt WolfGang Stammler, der Verfasser des entsprechenden Artikels in Trübners DeUtschem WörTERBUCH: „Es ist bedauerlich, daß das Wort, mit dem unsere Soldaten in den Tod stürmten, das ein Bestandteil der deutschen Heeressprache geworden ist, seinen Sinn im Volksmund verloren hat." (GöTZE 1939, 502).

Selbst wenn eine solche Verwendung an keiner Belegstelle nachzuweisen wäre, so ginge eine etymologische Angabe, die nur auf die heute für wahrscheinlich gehaltene (aber auch bereits im 19. Jahrhundert bisweilen vermutete) Übernahme aus der englischen Seemannssprache ${ }^{28}$ einginge, an zumindest einer „Wahrheit“ des 19. Jahrhunderts vorbei, und würde das korrekte Verständnis etwa der Stelle bei Arndt oder jeder anderen, an der ein Autor das Wort in bewusster Anspielung auf die Befreiungskriege verwendet, verhindern.

Nun kann man einwenden, dass ein Lexikograph keinen Stellenkommentar leisten kann und soll. Dem kann jedoch entgegengehalten werden, dass es sich hierbei nicht um die Erläuterung okkasioneller oder idiolektaler Phänomene handelt - und selbst um diese kommt der Lexikograph bisweilen nicht herum, wenn es ihm an geeigneteren Belegen fehlt - sondern oft um Wissen, das gewissermaßen mitlexikalisiert ist, und von jedem Sprecher abgerufen werden kann (,Kunst kommt von können“), und nicht erst vom Wortverwender, also in diesem Fall unserem Literaten, dem Wort angeheftet wird, wie das etwa bei einem Wortspiel der Fall ist. Auch geht es hier nicht um konkrete Artikelarbeit, sondern um methodische Überlegungen, deren Ziel es ist, historische Wortverwendungen so authentisch wie möglich zu beschreiben. Zudem ist etymologisches Wissen nicht nur für die literaturwissenschaftliche Interpretation einzelner Textstellen von Belang; sie kann viel weitreichendere Auswirkungen haben und damit auch lexikographisch relevant werden: So ließe sich z.B. ohne weiteres ein Fall vorstellen, wo die orthographische Trennung zweier Homophone auf eine falsche Etymologie gestützt wird.

Dass dies der Fall ist, kann anhand zweier zentraler Benutzungsweisen eines historischen Wörterbuchs leicht gezeigt werden. OsKar REICHMANN nennt als eine einer Reihe von „möglichen Benutzungsanlässen und Benutzerfragen“ des FWB (die natürlich ohne weiteres auch auf andere historische Wörterbücher übertragen werden können) die „Benutzung zur Behebung von Schwierigkeiten bei der Rezeption von Texten“. REICHMANN erläutert die entsprechende Situation:

„Ein an Texten des späten Mittelalters oder der frühen Neuzeit Interessierter hat Verständnisschwierigkeiten bei deren Lektüre. Dabei ist ,Verständnis' oberbegrifflich mindestens in doppeltem Sinne aufzufassen, einmal nämlich als pragmatisches und einmal als im engeren Sinne se-

28 Vermutlich hängt das Wort mit dem englischen Ausruf huzzah zusammen, der über die Seemanssprache als hurray ins Deutsche gekommen sein könnte (KLUGE 2002, 428, dort leider ohne zeitliche Eingrenzung und mit dem abschließenden Vermerk ,die Herkunft ist aber aufs ganze gesehen unklar" und PFEIFER 1995, 565).

Bereits Joachim Heinrich Campe plädierte für die Herkunft aus der Seemannssprache (CAmpe 1808,809 , s.v. Hurrah). Damit ist klar, dass auch diese Erklärung, die ja ebenfalls in ähnlicher Weise wie bei Arndt in manche Wortverwendung eingegangen sein mag, in einem entsprechenden Wörterbuchartikel nicht fehlen dürfte. Vgl. dazu den Beispielartikel ,hurra, hurrah“ am Ende dieses Beitrags. 
mantisches Verständnis. Der Lesende sucht seine Verständnisschwierigkeiten dadurch zu beheben, daß er im Wörterbuch diejenigen Wörter nachschlägt, die er offensichtlich überhaupt nicht versteht oder von denen er das Gefühl hat, sie unter bestimmten Aspekten, darunter auch Aspekten ihres Stellenwertes im Zusammenhang mit anderen Wörtern, nicht zu verstehen." (REICHMANN 1989, 26)

REICHMANN unterscheidet sodann „Wortlücken“, „Wortbedeutungslücken“29 und einige weitere Fälle, darunter den, in dem ,der Leser zwar das Wort und dessen im Text gültige Bedeutung ,irgendwie“ kennt, aber dennoch das Gefühl hat, einen bestimmten Bedeutungsaspekt nicht zu erfassen.“ (REICHMANN 1989, 27)

Eine zweite in unserem Zusammenhang besonders relevante Benutzungssituation ist die „Benutzung zur Behebung von Schwierigkeiten bei der Textproduktion“ (cf. REICHMANN 1989, 24-26). Hierbei geht es REICHMANN um Translationsprobleme, die beim Transferieren eines historischen Texts in heutiges Deutsch entstehen. ${ }^{30}$ Als Vermittler zwischen zwei Sprachvarietäten ist der „Übersetzer“ bzw. Überträger diejenige Person, die die hier diskutierten Probleme an deutlichsten zu spüren bekommt. Der Wörterbuchmacher wird daher bestrebt sein, nicht nur in den Bedeutungsangaben geeignete rezente Äquivalente anzubieten, sondern vor allem (aber nicht nur) dann, wenn diese die historische Bedeutung des (historischen) Explanandums nicht mit der gewünschten Genauigkeit zu „treffen“ scheinen, mit Hilfe weiterer Angaben dessen historische Verwendungsweise(n) besser zu beschreiben. Gerade in diese Benutzungssituation sind indirekt noch Dritte eingebunden, nämlich die Benutzer der Übersetzung oder Übertragung des in Frage stehenden Textes oder einiger Ausschnitte daraus z.B. in Kommentaren. Insbesondere letztere stellen eine für die Verwertung und Weitergabe lexikologisch erarbeiteter und lexikographisch aufgearbeiteter Daten prototypische Textsorte dar, d.h. sie sind über die reine Bedeutungsangabe hinaus angewiesen auf jede Form von Wissen über Wörter, das in einem Kommentar relevant werden könnte, umso mehr als eine breite linguistische historische Wortforschung abseits von Begriffsgeschichte und Lexikographie derzeit nicht existiert.

$\mathrm{Zu}$ den Aspekten, die ReIchmann erwähnt („,Der Lesende sucht seine Verständnisschwierigkeiten dadurch zu beheben, dass er im Wörterbuch diejenigen Wörter nachschlägt, [...] von denen er das Gefühl hat, sie unter bestimmten Aspekten [...] nicht zu verstehen"), können auch die genannten etymologischen Aspekte gehören, die, wie wir gesehen haben, ebenfalls in die Wortverwendung eingehen können, sei es auf denotativer (oben „referenzbezogen“ genannter) Ebene, sei es auf konnotativer Ebene (vgl. das Beispiel Friedhof). Tatsächlich dokumentiert das FWB solche volksetymologischen Phänomene, so etwa in dem auch in der „Lexikographischen Einleitung“ zitierten Beispielartikel „2abzucht":

,2abzucht [...]; aus lat. aquaeductus, aber im Frnhd. etymologisch undurchsichtig, deshalb starke Deformation der Form; als Gegentendenz dazu volksetymologische Formgebung, nach der die zweite Silbe überwiedegend zu -zucht, die erste tendenziell zu $a b$ - wird. Lautliche und morphologische Angleichung an $a b z u c h t$ ist wahrscheinlich. ${ }^{\text {“31 }}$

In diesen Zusammenhang, wenn auch in einen etwas weiter gesteckten Rahmen, gehört also auch das hier diskutierte Problem. Das Eingehen auf volketymologische Interpretationen ist

29 Terminologie nach WIEGAND 1977, 65.

$30 \mathrm{Zu}$ Reichmanns Verwendung der entsprechenden Terminologie vgl. ebda., S. 25, v.a. Anm. 18.

31 FWB, Artikel „2abzucht“, zitiert nach: Reichmann 1989, 82. 
ein Schritt in die Richtung, in die hier weitergedacht werden soll, es bleibt aber eine Angabe zur Etymologie eines Wortes und nicht eine Angabe zur Geschichte etymologischen Wissens über es.

\section{Methodische Überlegungen}

Das historische Bedeutungswörterbuch stellt eine sehr traditionsreiche Gattung innerhalb der deutschen Lexikographie dar. Umso erstaunlicher ist es, dass die hier beschriebenen Phänomene in ihrer lexikographischen Relevanz m.W. bislang nicht konsistent beschrieben wurden. Das historische Wörterbuch muss jedoch, mehr als alle anderen Wörterbücher, auf abweichendes historisches Wissen eingehen, wenn es Angaben zu Wortgeschichte und Etymologie macht, weil sich dies folgerichtig aus der Ausrichtung dieses Wörterbuchtyps ergibt.

Aufgrund dieser Situation soll im Folgenden eine methodische Fundierung versucht werden, die als Ausgangspunkt für weiterführende und spezifizierende Überlegungen auf diesem Gebiet dienen soll.

Eine Vorbemerkung ist an dieser Stelle unumgänglich: Es ist natürlich klar, dass die Zugänglichkeit solchen Wissens abhängig vom jeweils betrachteten Zeitraum erheblich variiert. Hier ist bislang implizit auf eine Zeit Bezug genommen worden, aus der etymologische Nachschlagewerke in größerem Umfang vorliegen, d.h., in der sich diese Textsorte im Sinne einer ,,modernen“ Lexikographie bereits etabliert hat, also etwa seit dem 18. Jahrhundert. Steht uns aus dieser Zeit eine Fülle von Wörterbüchern zur Verfügung, denen etymologisches Wissen entnommen werden konnte und kann, ${ }^{32}$ so nimmt die Zahl solcher Quellen ab, je weiter man sich von der Gegenwart entfernt. Zudem sind die entsprechenden Schriften unter Umständen schwer zugänglich. Da es hier jedoch nicht darum geht, das Aufsuchen, Einschätzen und Auswerten verschiedenster Quellen etymologischen Wissens unterschiedlichen Alters zu thematisieren, sondern lediglich darum, die Darstellung entsprechender Forschungsergebnisse methodisch zu fundieren, wird von einer Diskussion solcher Probleme in vollem Bewusstsein der Schwierigkeiten, die sich etwa für ein Wörterbuch zum Mittelhochdeutschen oder gar zum Althochdeutschen stellen würden, abgese-

32 In Bezug auf das 18, und 19. Jahrhundert sind zunächst die etymologischen Wörterbücher zur deutschen Sprache zu nennen, allen voran natürlich KLUGE 1883, FucHS 1898 oder HöFER 1815) oder auch das unter dem Pseudonym FrIedrich NorK herausgegebene ETYMOLOGISCHSYMBOLISCH-MYTHOLOGISCHE REAL-WÖRTERBUCH ZUM HANDGEBRAUCHE FÜR BIBELFORSCHER, ARCHÄOLOGEN UND BILDENDE KÜNSTLER (4 Bde., Stuttgart 1843-45). (Ergänzend könnten sodann die etymologischen Wörterbücher zu anderen indogermanischen Sprachen, wie etwa die von Franz Bopp, Georg Curtius, August Fick, Otto Böhtlingk und Rudolph Roth oder AuGUST Friedrich PotT hinzugezogen werden, die jedoch durch ihre spezifisch wissenschaftliche Ausrichtung einem nicht gerade etymologisch besonders interessierten Sprachteilhaber oder Sprachkünstler nicht bekannt gewesen sein dürften.)

Daneben können aber auch andere Wörterbücher, die keine eigentlichen etymologischen Wörterbücher sind, entsprechende Daten enthalten, wie z.B. die Wörterbücher von FRISCH, 1719 und 1741, oder KaltSCHMidT 1834. 
hen. ${ }^{33}$ Wir setzen also auch im Folgenden voraus, dass solche Quellen bereits gesichtet und ausgewertet wurden.

Dasselbe gilt für die zwangsläufig auftretenden Schwierigkeiten bei der Einschätzung der Verbreitung dieses Wissens. ${ }^{34}$ Das Auffinden entsprechender Nachweise stellt in den meisten Fällen für den Lexikographen eine kaum zu leistende Arbeit dar. Dieses grundsätzliche Problem lässt sich pragmatisch lösen, z.B. indem redaktionell festgelegt wird, dass der Nachweis des tatsächlichen Wissens eines Autors um eine bestimmte Etymologie durch den Lexikographen nicht erbracht werden muss. Es genügt, wenn die Möglichkeit, solches Wissen zu erwerben, unterstellt wird, und die abweichende Etymologie - soweit sie festgestellt werden konnte - ohne konkrete Bezugnahme auf eine zitierte Stelle ins Wörterbuch aufgenommen wird. Dies ist vor allem dann ausreichend, wenn sich die Erläuterungen des Lexikographen - wie in den meisten Fällen - auf Belegstellen verschiedener Autoren stützen.

Hinzu treten jedoch weitere methodische Probleme.

- Zum einen muss die Frage gestellt werden, bei welchen Wörtern man solche Anmerkungen machen möchte. Sicher sind diese bei Funktionswörtern weniger interessant als beim Kulturwortschatz. ${ }^{35}$ Auch können bestimmte Auswahlkriterien sinnvoll sein, wie z.B. das Vorkommen der fraglichen Wörter in bestimmten Texten bzw. Textsorten oder die Koppelung einer solchen Angabe an das Vorhandensein einer etymologischen Angabe (und damit an die Bedingungen derselben).

- Hinzu treten mikrostrukturbezogene methodische Fragen: An welcher Stelle ist eine entsprechende Angabe sinnvoll? Wie bindet man solche Angaben in die Wörterbuchstruktur ein, ohne dass die historischen Etymologien für rezente Forschungsergebnisse gehalten werden? Ist eine Kommentierung in jedem Fall verpflichtend?

Es ist sinnvoll, bei dem ersten Problem vom damaligen Wortbenutzer auszugehen. Sind für diesen beispielsweise durch das vom rezenten abweichende etymologische Wissen bestimmte, von den rezenten differierende Möglichkeiten der Wortverwendung feststellbar, so muss dies ins Wörterbuch aufgenommen werden, da mit einer „Mitlexikalisierung“ dieser Möglichkeiten zu rechnen ist.

33 Zum Umgang mit Etymologie im Mittelalter vgl. KLINCK 1970, RuberG 1975, 267-330, sowie OHLY 1966.

Vgl. zum gar nicht so verschiedenen Umgang mit Etymologie um 1800 beispielhaft die Arbeiten über Johann ARnold Kanne, in jüngster Zeit etwa Willer 2000, 113-129. Weiterführende Literatur dort.

34 In jüngerer Zeit finden sich womöglich Äußerungen über die Benutzung von Nachschlagewerken, vgl. etwa die vielen Äußerungen von Zeitgenossen zum Adelung (hierzu u.a. HenNe 1984, 98108), bisweilen gibt es sogar Belege für die Beschäftigung mit etymologischen Fragen, wie hier in GoETHES „Faust“:

„GREIF schnarrend. Nicht Greisen! Greifen! - Niemand hört es gern, / Daß man ihn Greis nennt. Jedem Worte klingt / Der Ursprung nach, wo es sich her bedingt: / Grau, grämlich, griesgram, greulich, Gräber, grimmig, / Etymologisch gleicherweise stimmig, / Verstimmen uns.

MEPHISTOPHELES. Und doch, nicht abzuschweifen, / Gefällt das Grei im Ehrentitel Greifen. GREIF wie oben und immer so fort. Natürlich! Die Verwandtschaft ist erprobt, / Zwar oft gescholten, mehr jedoch gelobt; / Man greife nun nach Mädchen, Kronen, Gold, / Dem Greifenden ist meist Fortuna hold."

(GoETHE/SCHÖNe 1994, 288, Vers 7093-7103.)

35 Doch sollten nicht a priori bestimmte lexikalische Gruppen ausgeschlossen werden. Wie das Beispiel Hurra(h) gezeigt hat, sind es nicht nur die diskursprägenden Begriffe wie Gott oder deutsch, die in dieser Hinsicht von Interesse sein können. 
Der zweite Problemkomplex ist unabhängig von der jeweiligen Wörterbuchstruktur nicht sinnvoll zu lösen. Die Art und Weise, wie historisches etymologisches Wissen im Wörterbuch repräsentiert ist, hängt von der Anlage und den Zielen des jeweiligen Wörterbuchs ab. Ob solches Wissen für das gesamte semantische Spektrum eines Wortes oder nur für bestimmte Einzelbedeutungen von Belang ist, oder ob der Lexikograph es gar nur in einer Kommentierung einer einzelnen, stärker idiolektal geprägten Belegstelle unterbringen möchte, ist vermutlich sogar nur für jeden einzelnen Wortartikel jeweils neu zu entscheiden. Daher ist die Übernahme eines Programms zur Erstellung etymologischer Angaben, wie etwa PATrick Dockar Drysdale es vorgestellt hat, ${ }^{36}$ ohne Modifikationen weder für diesen Angabentyp noch für jedes Wörterbuchprojekt möglich. Sicher ist eine Kommentierung der Differenz von aktuellem etymologischem Wissen grundsätzlich sinnvoll und notwendig, obwohl sich auch andere (schlechtere) Möglichkeiten denken lassen, wenn z.B. ein grundsätzlicher Benutzungshinweis formuliert wird oder (besser) durch klare Formulierungen oder besondere typographische Auszeichnung hinreichend auf die Besonderheit solcher etymologischer Angaben hingewiesen wird.

Es kann jedoch zusammenfassend festgehalten werden, dass - jeweils modifiziert durch die Anlage, die Zielgruppe und die inhaltlichen Ausrichtung des jeweiligen Wörterbuchs Überlegungen zu den folgenden Fragen immer angestellt werden müssen:

1. Welchen Zweck sollen die Angaben zu historischen Etymologien im Wörterbuch erfüllen?

2. Bei welchen Wörtern sollen solche Anmerkungen gemacht werden?

3. An welcher Position im Artikel ist eine entsprechende Angabe sinnvoll?

4. Kann bzw. soll ein Nachweis des tatsächlichen Wissens eines Autors um eine bestimmte Etymologie durch den Lexikographen erbracht werden? (Dies kann bei Autorenwörterbüchern oder bei einzelnen Textstellen durchaus nicht ganz unsinnig bzw. unmöglich sein)

5. Welchen Komplexitätsgrad sollen diese Angaben (etwa in Bezug auf die Schilderung einer zeitgenössischen Diskussion, Literaturhinweise, eigene Stellungnahmen des Lexikographen o.ä.) haben?

6. Ist die historische Etymologie in Bezug auf den Belegzeitraum unumstritten gewesen?

7. Wie viele/welche konkurrierenden Etymologien sollen ggf. aufgenommen werden?

8. Auf welche Weise werden die Angaben zu historischen Etymologien von rezenten Forschungsergebnissen zur Etymologie abgesetzt?

9. Ist eine Kommentierung in jedem Fall verpflichtend?

Eine Mindestlösung, etwa für ein einbändiges historisches Bedeutungswörterbuch, könnte in etwa folgendermaßen aussehen:

1. Welchen Zweck sollen die Angaben zu historischen Etymologien im Wörterbuch erfüllen? Angaben zu historischen Etymologien haben ausschließlich den Zweck, mögliches etymologisches Wissen der Autoren (und/oder der zeitgenössischen Leser) dieser Texte explizit zu machen, das eine durch dieses Wissen erst realisierbare differente Wortverwendung ermöglichte.

2. Bei welchen Wörtern sollen solche Anmerkungen gemacht werden?

36 Basierend auf einer früheren Publikation stellt DrYSDALE sein für das Englische erstellte Programm in seinem Aufsatz „Etymological Information in the General Monolingual Dictionary“ vor: „(1) source language or language family; (2) first English form and/or immediate source; (3) date or period of entry into English; (4) changes of form and meaning in English; (5) intermediate stages, pre-English; (6) ultimate known source; (7) semantic development; (8) ultimate underlying formm known or hypothetical; (9) cognates in related languages; (10) other English words from the same base“. DRYSDALE 1989, 525-530, Zitat S. 527.

Vgl. hierzu auch DRYSDALE o.J., 39-50. 
Bei Wörtern, deren Artikel (gebunden an bestimmte, z.B. semantisch begründete Bedingungen) eine etymologische Angabe enthalten, wird geprüft, ob sich das rezente etymologische Wissen so vom historischen Wissen unterscheidet, dass man feststellen muss, dass zwei verschiedene Etymologien vorliegen, und nicht etwa die jüngere nur eine verfeinerte Variante der älteren darstellt. Dann wird anhand der Belege geprüft, ob das abweichende etymologische Wissen des Belegzeitraums bestimmte, von den rezenten differierende, also vor allem eingeschränkte oder erweiterte Wortverwendungen bedingte, die in ihrem etymologischen Zusammenhang eventuell vom Verfasser einer Belegstelle gewusst oder von deren zeitgenössischen Lesern verstanden wurden. In solchen Fällen werden Angaben zu historischen Etymologien gemacht.

3. An welcher Position im Artikel ist eine entsprechende Angabe sinnvoll?

Die Angaben zu historischen Etymologien werden im Zusammenhang mit den etymologischen Angaben gemacht und sprachlich eindeutig als solche gekennzeichnet. Entscheidend für die Reihenfolge der beiden Angaben ist die flüssige Lesbarkeit der jeweiligen Formulierung. ${ }^{37}$

4. Soll der Nachweis des tatsächlichen Wissens eines Autors um eine bestimmte Etymologie durch den Lexikographen erbracht werden?

Nein. Es genügt die Feststellung eines größeren Verbreitungsgrades (etwa in Form erschwinglicher kommerzieller etymologischer Wörterbücher) und damit der Zugänglichkeit der historischen Etymologie. Dies muss natürlich in den Benutzerhinweisen vermerkt werden. Eine klar etymologisierende Verwendung wird allerdings explizit als solche gekennzeichnet.

5. Welchen Komplexitätsgrad sollen diese Angaben haben?

Der Komplexitätsgrad solcher Angaben ist natürlich stark zielgruppenabhängig. Richtet sich das Wörterbuch an ein breites Publikum, bedeutet dies unter Umständen, dass Angaben zur Etymologie nur insoweit gemacht werden, als sie zur Bedeutungsbeschreibung eines Wortes beitragen. ${ }^{38}$ In diesem Fall werden dann auch Angaben zu historischen Etymologien knapp und leicht verständlich gehalten. Weiterführende Literaturhinweise werden nur in Ausnahmefällen gegeben. ${ }^{39}$ Auch die Aufnahme konkurrierender etymologischer Erklärungen wird in diesem Fall nur in sehr eingeschränktem Rahmen ${ }^{40}$ möglich sein.

6. Ist die historische Etymologie in Bezug auf den Belegzeitraum unumstritten gewesen?

Wenn nicht, wird dies im Artikel zumindest erwähnt (etwa durch Hinweise wie „vielleicht“, „wahrscheinlich“ oder „,verbreitet"), die konkurrierenden Etymologien werden aber nur dann aufgenommen, wenn ihre Kenntnis für das Verständnis des jeweiligen Wortes hilfreich oder notwendig sind.

7. Wie viele/welche konkurrierenden Etymologien sollen aufgenommen werden?

S. die Punkte 5 und 6.

8. Wie werden die Angaben zu historischen Etymologien von rezenten Forschungsergebnissen zur Etymologie getrennt?

Die Trennung erfolgt aus Gründen der Lesbarkeit durch eindeutige sprachliche Markierung: z.B. „,zeitgenössisch“vs. „rezent“, „heute“ vs. ,im Belegzeitraum“ und Ähnliches.

9. Ist eine Kommentierung in jedem Fall verpflichtend?

37 Vgl. hierzu die Beispiele aus dem KLASSIKERwöRTERBUCH weiter unten.

38 Vgl. hierzu MaLKIEL 1990, 1326.

39 Schon ein einbändiges etymologisches Wörterbuch kann sich aus Platzgründen ausführliche Literaturhinweise nicht leisten, umso weniger ein Wörterbuch, dessen primäres Erklärungsinteresse nicht auf diesem Gebiet liegt. Vgl. hierzu MaLKIEL 1990, 1325.

40 Dieser orientiert sich an der Zugänglichkeit der entsprechenden Informationen sowie an der Konsensfähigkeit der Vorschläge für die Zeitgenossen. Mehrheitlich als absurd oder abwegig abgelehnte Vorschläge werden nicht aufgenommen (Vgl. hierzu Peter Wunderlis Aufsatz „Sprachursprungstheorien in der Romania", in dem er auch für solche Reaktionen Beispiele anführt, bes. S. 194). Maßgeblich für die Zeit um 1800 wären etwa die beiden großen Wörterbücher ADELUNGS und CAMPES, da vor allem ersterer einerseits einen umfassenden Überblick über das vorwissenschaftliche etymologische Wissen seiner Zeit gibt (vgl. hierzu PfeIfER 1984, 238) und andererseits nachweislich von vielen Korpusautoren - in unterschiedlichem Maße und bisweilen äußerst kritisch - benutzt wurden (vgl. hierzu u.a. Henne 1984, 98-108.). 
Der Lexikograph hat den Freiraum, aufgrund der Beleglage und seines um die Etymologie des Wortes versammelten Wissens die Entscheidung für oder gegen eine weiterführende Kommentierung in jedem Einzelfall eigenständig zu treffen.

\section{Beispiele}

Im Folgenden soll anhand einiger ausgewählter Beispiele der Umgang mit diesem Angabentyp im KLASSIKERWÖRTERBUCH (cf. BRÜCKNER/KNOOP 2003) dokumentiert werden. Gleichzeitig können diese als Vorschläge für den Umgang mit solchen lexikographischen Daten betrachtet werden. ${ }^{41}$

hurra, hurrah. Interj. [ $\left.38^{58}\right]$ Die genaue Wortgeschichte ist ungewiß. Ein möglicher Zusammenhang von mit "hurren" (vgl. Thurren und $\nearrow$ hurre) ist umstritten. Im Falle einer Verwandtschaft könnte die durch mhd. „hurren” (vgl. lat. „currere” ,laufen") ausgedrückte schnelle Bewegung auch eine Rolle für die Bedeutung von „hurra” spielen. Eventuell hängt das Wort aber auch mit dem englischen Ausruf "huzzah" zusammen, der über die Seemanssprache als "hurray” ins Deutsche gekommen sein könnte. Obwohl bereits Campe für die Herkunft aus der Seemannssprache plädiert (Ca II/809), und das Wort schon im 18. Jh. (Vgl. Bürger, Lenore (FA 684) 1773) und sogar im Mhd. (als "hurrâ") belegt ist, scheint das 19. Jahrhundert den Ausruf mit den Befreiungskriegen in Verbindung bringen zu wollen. Man hielt eine Verwandtschaft mit russisch/tatarisch „urá" ,schlag drein für wahrscheinlich: dieser Schlachtruf sei von den russischen Soldaten übernommen worden und in den Befreiungskriegen zum Schlachtruf der deutschen Heere geworden: „Hurra, ihr Kamraden! Hurra, ihr Soldaten! Hurra! ist Franzosen ein tödlicher Klang!" (Arndt/Leffson, Arndts Werke 1/102). Mit dieser Assoziation ist während des ganzen 19. Jahrhunderts zu rechnen. Auch Trübner verzeichnet diese Bedeutung noch (III/501f: „das Wort, mit dem unsere Soldaten in den Tod stürmten”). [...]

In diesem Fall wird zunächst der heutige Wissensstand referiert, („Der Zusammenhang von „hurra“ mit „hurren“ [...] als „hurray“ ins Deutsche gekommen sein könnte.“). Danach geht der Lexikograph unter Verwendung des bereits angesprochenen Arndt-Zitats auf die Verhältnisse im 19. Jahrhundert ein („Auch wenn Campe [...] „Hurra, ihr Kamraden! Hurra, ihr Soldaten! Hurra! ist Franzosen ein tödlicher Klang!“ (ARndT/LefFson 1912, I/102).) Der letzte Satz bestätigt die Festigung der älteren Auffassung und markiert gleichzeitig das Ende ihrer Geltung.

Ganz ähnlich im folgenden Beispiel:

Fiaker. m. [3] Aus dem frz. „fiacre”. Über die Herkunft des Wortes gibt es verschiedene Theorien, von denen eine im 18. und 19. Jh. sehr weit verbreitete hier zusammengefaßt werden soll: Mitte des 17. Jh. gründete Nicolaus Sauvage in Paris ein Mietkutschenunternehmen, das seinen Stammsitz in einem großen Gebäude mit Hof und Stallungen hatte, und nach einem heiligen schottischen König „Hôtel de Saint Fiacre“ genannt wurde. Der Name des Gebäudes übertrug sich im Laufe der Zeit auf die Mietkutschen. Heute ist gesichert, daß es sich bei dem genannten Heiligen Fiacrius nicht um einen schottischen König, sondern um einen irischstämmigen Einsiedler aus dem 7. Jh. handelte, der auf einem Grundstück, das inm von Bischof Faro von Méaux zur Verfügung gestellt wurde, eine Einsiedelei gründete, die sich später zu einem Kloster entwickelte. Ob das Gebäude wirklich „Hôtel de Saint Fiacre" hieß und der Name daher rührt oder ob die Verbindung zum HI. Fiacrius über andere Wege hergestellt wurde, bleibt allerdings unklar. [...]

41 Bei dieser Zahl handelt es sich um eine Belegzahlangabe. 
Eine befriedigende Erklärung der Herkunft von Fiaker ist bis heute nicht gelungen. Auf diese Lage wird zunächst aufmerksam gemacht („Über die Herkunft des Namens gibt es verschiedene Theorien"), danach wird ein im 18. und 19. Jahrhundert weithin bekannter Erklärungsversuch referiert.

Ein letztes Beispiel bietet der Artikel „topp“:

topp. Interj. [22] Wahrscheinlich aus der altniederdeutschen Rechtssprache stammender Ausdruck, der als Ausruf die die Rechtsgültigkeit bekräftigende Berührung (Handschlag oder Zusammenhalten der Daumen) begleitete. Etymologisch ist das Wort vermutlich verwandt mit den niederdeutschen Formen dopp(e), dip, tippen und stip, die das (wiederholte) Berühren, Eintauchen oder den Punkt (einer Berührung) bezeichnen (Vgl. heutiges „tupfen“, engl. „to dip“). Die in den Wörterbüchern des 18. und 19. Jh. behauptete Entlehnung aus dem frz. ,je tope (et tingue)“, etwa ,ich will beim Spielen mithalten' (vgl. etwa Ad IV, 630), findet sich in den modernen etymologischen Nachschlagewerken nicht mehr. [...]

Hier nimmt die Darstellung des rezenten Wissensstandes den größten Raum ein. Die im Belegzeitraum verbreitete Herleitung aus dem Französischen ,,je tope (et tingue)“ wird nur ergänzend angefügt.

\section{Literatur}

Abaev 1980 = Abaev, Vasilij Ivanovi: Die Prinzipien eines etymologischen Wörterbuchs. Deutsch von Heinz Dieter Pohl. In: Mayrhofer, Manfred: Zur Gestaltung des Etymologischen Wörterbuches einer „Großcorpus-Sprache“. Mit je einem Anhang von VasiLis Ivanovi Abaev sowie von Karl Hoffmann und Eva Tichy (= Österreichische Akademie der Wissenschaften. Philosophisch-historische Klasse,. Sitzungsberichte, 368. Band. Veröffentlichungen der Kommission für Linguistik und Kommunikationsforschung. Herausgegeben von MARIO WANDRUSZKA und Wolfgang U. Dressler. Heft 11). Wien 1980, 29-46.

ANTOS 1996 = ANTOS, Gerd: Laien-Linguistik. Studien zu Sprach- und Kommunikationsproblemen im Alltag. Am Beispiel von Sprachratgebern und Kommunikationstrainings. Tübingen 1996.

Arndt/LefFson 1912 = LefFson, August (Hrsg.): Arndts Werke. Auswahl in zwölf Teilen. Herausgegeben mit Einleitungen und Anmerkungen versehen von August LefFson. Band: I, Gedichte. Mit einem Lebensbild von Wilhelm StefFens. Berlin/Leipzig. Wien. Stuttgart 1912.

Augst 1975 = AugSt, GerHARD: Überlegungen zu einer synchronen etymologischen Kompetenz. In: Augst, Gerhard: Untersuchungen zum Morpheminventar der deutschen Gegenwartssprache. Tübingen 1975, 156-230.

BALDINGER 1973 = BALDINGER, KuRT: Zum Einfluß der Sprache auf die Vorstellungen des Menschen. (Volksetymologie und semantische Parallelverschiebung). Vorgetragen am 11. November 1972 (= Sitzungsberichte der Heidelberger Akademie der Wissenschaften, Philosophischhistorische Klasse, Jahrgang. 1973, 2. Abhandlung). Heidelberg 1973.

Bartels 1996 = Bartels, Klaus: Wie Berenike auf die Vernissage kam. 77 Wortgeschichten. Darmstadt 1996.

Baumann 1967 = Baumann, Hans-Heinrich: Sekundäre Motivationen bei romanischen Tierbezeichnungen. (Blindschleiche, Hornisse, Wiesel, Bachstelze, Schleiereule). Bonn 1967.

Bebermeyer 1974 = Bebermeyer, Renate: Zur Volksetymologie: Wesen und Formen. In: MöCKelmann, Jochen (Hrsg.): Sprache und Sprachhandeln. Festschrift für Gustav Bebermeyer zum 80. Geburtstag am 16. 10. 1970. Hildesheim.New York 1974, 156-187. 
Bloch/von Wartburg 1964 = Bloch, Oscar/Walther von Wartburg: DictionNaire ÉtymoLOGIQUE DE LA LANGUE FRANÇAISE. 4. édition revue et augmentée par Walther von Wartburg, Paris 1964.

BRÜCKNER/KNOOP 2003 = BRÜCKNER, DOMINIK/UlRICH KNOOP: Das Klassikerwörterbuch. In: Zeitschrift für Germanistische Linguistik 31. 2003, 62-86.

BÜrger/HäntZSChEl 1987 = Bürger, GotTfried August: Sämtliche Werke. Herausgegeben von GüNTER und Hiltrud HäNTZSCHEL. München. Wien 1987.

Campe 1808 = Campe, Joachim Heinrich: Wörterbuch der deutschen Sprache. Bd. II, $F-K$, Braunschweig 1808.

DeEg 1995 = Deeg, Max: Die altindische Etymologie nach dem Verständnis Yâska's und seiner Vorgänger. Eine Untersuchung über [sic] ihre Praktiken ihre literarische Verbreitung und ihr Verhältnis zur dichterischen Gestaltung und Sprachmagie (= Würzburger Studien zur Sprache \& Kultur. Indologie. Vergleichende Sprachwissenschaft, Bd. II). Dettelbach 1995.

Drysdale, o.J. = Drysdale, Patrick Dockar: Dictionary Etymologies. What? Why? And for Whom? In: Michell, Gillian (Hrsg.): Papers of the Dictionary Society of North America 1979. London Ontario, o.J., 39-50.

Drysdale, 1989. = Drysdale, Patrick Dockar: Etymological Information in the General Monolingual Dictionary. In: Hausmann, Franz Josef [u.a.] (Hrsg.): Wörterbücher. Ein internationales Handbuch zur Lexikographie. 1. Teilband (Handbücher zur Sprach- und Kommunikationswissenschaft, Bd. 5.1). Berlin. New York 1989, 525-530.

DWB = Grimm JaCoB/Wilhelm Grimm: Deutsches WöRterbuCh, Leipzig 1854-1960 [16 Bände in 32 Teilbänden] (Fotomechanischer Nachdruck, München 1984).

FÖRSTEMANN 1852 = FöRSTEMANN, ERNST: Ueber deutsche volksetymologie. In: Zeitschrift für vergleichende Sprachforschung 1 (1852), 1-25.

Frisch 1719 = Frisch, Johann Leonhard: Nouveau Dictionaire des Passagers FrançoisAllemand et Allemand-François, Oder neues Frantzösisch-Teutsches und TeutschFrantzösisches WÖrter-Buch. WOrinnen Alle Frantzösische Wörter, AUCH DER KÜNSTE UND WiSSENSCHAFFTEN, AUS DEN VOLLKOMMENSTEN UND NEUESTEN DiCTIONARIIS, NEBST DEN NÖTHIGSTEN CONSTRUCTIONEN UND REDENS-ARTEN, DURCH KÜRTZLICH GEZEIGTE ETYMOLOGIE, UND DURCH DAS GEBRÄUCHLICHSTE AUCH REINESTE TEUTSCHE ERKLÄRET WORden; Im Teutschen Theile aber eine so grosse Verbesserung und Vermehrung geschehen, dass die Liebhaber beyder Sprachen dieses Buch mit grossem Nutzen GEBRAUCHEN KÖNNEN. Andere Auflage, Leipzig 1719.

Frisch 1741 = Frisch, Johann Leonhard: Teutsch-Lateinisches Woerter-Buch. Darinnen NICHT NUR DIE URSPRUENGLICHEN, NEBST DENEN DAVON HERGELEITETEN UND ZUSAMMENGESETZTEN ALLGEMEIN GEBRAEUCHLICHEN WOERTER; SONDERN AUCH DIE BEY DEN MEISTEN Kuensten und Handwerken, bey Berg- und Saltzwerken, Fischereyen, Jagd-, Forstund Hauss-Wesen, U.A.M. Gewoehnliche Teutsche Benennungen Befindlich, Vor ALLen, Was NOCh IN Keinem Woerter-Buch geschehen, Denen Einheimischen und AusLAENDERn, SO DIE IN DEN mitTlern Zeiten geschriebenen Historien, Chroniken, UeberSETZUNGEN, REIMEN U.D.G. MIT IHREN VERALTETEN WOERTERN UND AUSDRUECKUNGEN VERSTEHEN WOLLEN, MOEGLICHST ZU DIENEN; Mit UEBERALl BEyGESETZTER NOETHIGEN ANFUeHRUNG Der Stellen, wo Dergleichen in DEN Buechern ZU finden, Samt angehaengter THEILS VERSICHERTEN, THEILS MUTHMASSLICHEN ETYMOLOGIE UND CRITISCHEN ANMERKUNgen; Mit allem Fleiss viel Jahr ueber zusammengetragen Und Jetzt den Gelehrten Zur beliebigen Vermehrung und Verbesserung ueberlassen. Nebst einem Register der LATEINISCHEN WOERTER. 2 Bde., Berlin 1741.

Fuchs 1898 = Fuchs, Paul Immanuel: Deutsches Wörterbuch auf etymologischer GrundLAGE. Mit Berücksichtigung wichtigerer Mundart- und Fremd-Wörter sowie vieler Eigennamen. Stuttgart 1898.

GÄRTNER 1996 = GÄRTNER, KuRT: Frau und Freude (vrouwe und vröide). Etymologisieren in der mittelhochdeutschen Literatur. In: Herrmann, Michael/Hölz, KarL (Hrsgg.): Sprachspiele und Sprachkomik - Jeux de mots et comique verbal (= Trierer Studien zur Literatur 29). Frankfurt/M 1996, 35-52. 
Gauger 1994 = Gauger, Hans-Martin: „Der etymologische Holzweg“. In: park Bd. XVIII, H. 47. 1994, 23-39.

Genius, o.J. = Genius, Adolf: Neues grosses Fremdwörterbuch. Erster Teil. $A-K$. Regensburg o.J.

Goethe/Schöne 1994 = Schöne, Albrecht (Hrsg.): Johann Wolfgang Goethe: Faust. Texte (= Johann WolfGang Goethe: Sämtliche Werke. Briefe, Tagebücher und Gespräche. I. Abteilung: Sämtliche Werke. Band 7/1). Frankfurt am Main 1994.

Götze 1993 = Götze, Alfred (Hrsg.): Trübners deutsches Wörterbuch. Bd. 3, G - H, Berlin 1939.

GuralniK et al. 1979 = Guralnik, David Bernard et al. (Hrsg.): Webster's New World DictiONARY. $2^{\text {nd }}$ college edition. Cleveland 1970.

Henne 1984 = Henne, Helmut: Johann Christoph Adelung - Leitbild und Stein des Anstoßes. Zur Konstitutionsproblematik gegenwartsbezogener Sprachforschung. In: BAHNER, WERNER (Hrsg.): Sprache und Kulturentwicklung im Blickfeld der deutschen Spätaufklärung. Der Beitrag Johann Christoph Adelungs (= Abhandlung der sächsischen Akademie der Wissenschaften zu Leipzig. Philolog.-hist. Klasse. Bd. 70, H. 4). Berlin 1984, 98-108.

Hettrich 1993 = Hettrich, Heinrich: Deutsche Etymologie bei Lexer und heute. In: Brunner, HoRst (Hrsg.): Matthias von Lexer. Beiträge zu seinem Leben und Schaffen (= Zeitschrift für Dialektologie und Linguistik. Beihefte, Heft 80). Stuttgart 1993, 133-140.

HöFer 1815 = Höfer, Matthias: Etymologisches Wörterbuch der IN Oberdeutschland, VORZÜGLICH ABER IN OESTERREICH ÜBLICHEN MUNDART. 3 Bde., Linz 1815.

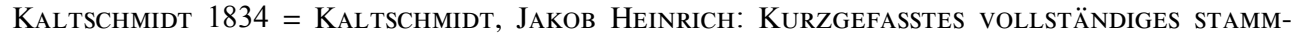
UND SINNVERWANDTSCHAFTliches GeSAMmT-WÖrTERbUCH DER DEUTSCHEN SPRACHE AUS ALLen ihren Mundarten und mit allen Fremdwörtern. Ein Hausschatz der MutterspraCHE FÜR ALle StÄNDE DES DeUtSChEN VOLKes [...]. Leipzig 1834.

KäSTNER 1991 = KäSTNER, HANNES: „Der grossmächtige Riese und Recke Theuton“: Etymologische Spurensuche nach dem Urvater der Deutschen am Ende des Mittelalters. In: Deutsche Philologie 110. H. 1, 1991, 68-97.

KIRWIN 1985 = Kirwin, William: Folk Etymology: Remarks on Linguistic Problem-Solving and who does it. In: Lore and Language IV. H. 2. 1985, 18-24.

KlincK 1970 = Klinck, Roswitha: Die lateinische Etymologie des Mittelalters (= Medium Aevum. Philologische Studien 17). München 1970.

Kluge 1883 = Kluge, Friedrich: Etymologisches Wörterbuch der deutschen Sprache. Straßburg 1883.

Kluge 2002 = Kluge, Friedrich: Etymologisches Wörterbuch der Deutschen Sprache. Bearbeitet von Elmar Seebold. 24., durchgesehene und erweiterte Auflage. Berlin 2002.

LeXer 1872 = LeXer, Matthias von: MitTelhochdeutsches HaNdwörterbuch. Zugleich als SUPPLEMENT UND ALPHABETISCHER INDEX ZUM MitTELHOCHDEUTSCHEN WÖRTERBUCHE VON BENECKE-MÜLlER-ZARNCKE. Bd. 1, $A-M$, Leipzig 1872.

Lindsay 1911 = Lindsay, Wallace Martin (Hrsg.): Isidori Hispalensis Episcopi Etymologiarum sive originum libri XX. Oxford 1911.

Looff 1900 = LoOff, Friedrich Wilhelm: Allgemeines Fremdwörterbuch [...]. Fünfte Auflage. Besorgt von Dr. Fr. Ballauf. Langensalza 1900.

MALKIEL 1990 = MALKIEL, YAKOv: Das etymologische Wörterbuch von Informanten- und Korpussprachen. In: Hausmann, Franz Josef (Hrsg.): Wörterbücher. Ein internationales Handbuch zur Lexikographie, Bd. II (= Handbücher zur Sprach- und Kommunikationswissenschaft, Bd. 5.2). Berlin. New York 1990, 1323-1334.

NORK 1843-1845 = NORK, FRIEDRICH: ETYMOLOGISCH-SYMBOLISCH-MYTHOLOGISCHES REAL-

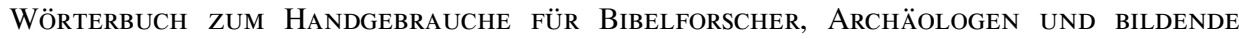
KÜNSTLER. 4 Bde.. Stuttgart 1843-1845.

Ohly 1966 = Ohly, Friedrich: Vom geistigen Sinn des Wortes im Mittelalter (= Libelli 218). Sonderausgabe, unveränderter reprographischer Nachdruck aus: Zeitschrift für deutsches Altertum und deutsche Literatur 89 (1958/59). Darmstadt 1966.

Olschansky 1996 = Olschansky, Heike: Volksetymologie (= Reihe Germanistische Linguistik 175). Tübingen 1996. 
Paul 1992 = Paul, Hermann: Deutsches Wörterbuch. 9., vollständig neu bearbeitete Auflage von Helmut Henne und Georg Objartel unter Mitarbeit von Heidrun KämPer-Jensen. Tübingen 1992.

Pfeifer 1984 = PFeIfer, Wolfgang: Adelungs Stellung zur Etymologie in seinem Wörterbuch. In: BAHner, Werner (Hrsg.): Sprache und Kulturentwicklung im Blickfeld der deutschen Spätaufklärung. Der Beitrag Johann Christoph Adelungs (= Abhandlung der sächsischen Akademie der Wissenschaften zu Leipzig. Philolog.-hist. Klasse. Bd. 70, H. 4). Berlin 1984, 233-238.

Pfeifer 1995 = Pfeifer, Wolfgang: Etymologisches Wörterbuch Des Deutschen. Ungekürzte, durchgesehene Ausgabe. München 1995.

von Polenz 1980 = Polenz, Peter von: Wortbildung. In: Althaus, Hans Peter/Helmut HenNe/Herbert ERnst Wiegand (Hrsg.): Lexikon der Germanistischen Linguistik. 2., vollständig neu bearbeitete und erweiterte Auflage. Tübingen 1980, 169-180.

ReICHMANn 1989 = ReICHMANn, OSkar, Lexikographische Einleitung. In: ANDERSON, RoberT R./Ulrich Goebel (Hrsg.): Frühneuhochdeutsches Wörterbuch. Band I, Einführung, a äpfelkern. Bearbeitet von OsKar Reichmann. Berlin 1989.

Ruberg 1975 = Ruberg, Uwe: Verfahren und Funktionen des Etymologisierens in der mittelhochdeutschen Literatur. In: Fromm, Hans/Wolfgang Harms/Uwe Ruberg (Hrsg.): Verbum et Signum. Bd. I: Beiträge zur mediävistischen Bedeutungsforschung. München 1975, 267-330.

WDG = KLAPPENBACH, RUth/STEINITZ, WolFGANG: Wörterbuch der deutschen Gegenwartssprache. 6 Bände, 8., bearbeitete Auflage. Berlin 1977ff.

Weisgerber 1957 = Weisgerber, Leo: Die Muttersprache im Aufbau unserer Kultur (= Von den Kräften der deutschen Sprache, Bd. III). 2., erweiterte Auflage. Düsseldorf 1957.

Welzig 1999 = Welzig, Werner (Hrsg.): Wörterbuch DER RedenSARTEN Zu DER VON Karl Kraus 1899 bis 1936 herausgegebenen Zeitschrift „Die Fackel“. Wien 1999.

Werz 1945 = Werz, ElFRIEDE: Der Ohrwurm. Diss. Marburg 1945.

WIEGAND 1977 = WiEgAND, HERBERT ERNST: Einige grundlegende semantisch-pragmatische Aspekte von Wörterbucheinträgen. Ein Beitrag zur praktischen Lexikologie. In: Kopenhagener Beiträge zur germanistischen Linguistik 12. 1977, 59-149.

Willer 2000 = Willer, Stefan: „übersetzt: ohne Ende“. Zur Rhetorik der Etymologie bei Johann Arnold Kanne. In: Jaeger, Stephan/Stefan Willer: Das Denken der Sprache und die Performanz des Literarischen um 1800 (= Stiftung für Romantikforschung, Bd. X). Würzburg 2000. $113-129$.

Wunderli 1994a = Wunderli, Peter (Hrsg.): Herkunft und Ursprung. Historische und mythische Formen der Legitimation. Sigmaringen 1994.

Wunderli 1994b = Wunderli, Peter: Sprachursprungstheorien in der Romania. In: Wunderli, Peter (Hrsg.): Herkunft und Ursprung. Historische und mythische Formen der Legitimation. Sigmaringen 1994, 179-205. 